\title{
Transmission Electron Microscopy Study of FeHfN Thin Films for Magnetic Properties Optimization and Integration Above Silicon Circuits.
}

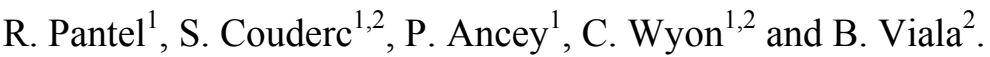 \\ 1) STMicroelectronics, 850 Rue Jean Monnet, F-38926 Crolles, France \\ 2) LETI, CEA Grenoble, 17 rue des Martyrs, F-38054 Grenoble, France
}

Integration of miniaturized magnetic devices is one of the major challenges for the development of system on chip (SoC). For instance the introduction of inductors above Integrated Circuits (IC) requires the use of soft magnetic materials exhibiting high permeability (high saturation magnetization) at high frequency $(1-10 \mathrm{GHz})$ [1-2]. We present a detailed microscopy study of PVD FeHfN films in order to understand and optimize their magnetic properties. Their nano-crystalline structure is studied using TEM dark field imaging, high resolution TEM (HRTEM) and micro-diffraction. Their chemical composition is analyzed at the nanometer scale using Energy Filtered TEM (EFTEM) and Scanning TEM combined with Electron Energy Loss Spectroscopy (STEM-EELS). Magnetic properties are measured using a vibrating sample magnetometer (VSM).

Experimental details: FeHfN films were deposited by reactive $\mathrm{RF}$ diode sputtering using a $\mathrm{Fe}_{97.6} \mathrm{Hf}_{2.4}$ target in a mixed $\mathrm{Ar}+\mathrm{N}_{2}$ atmosphere. The films were grown on oxidized (100) silicon wafer with a 100 Oe magnetic field applied in the plane of the growing film. The nitrogen content was varied by changing the $\mathrm{N}_{2} /\left(\mathrm{Ar}+\mathrm{N}_{2}\right)$ gas flow rate and its final concentration was measured by nuclear reaction analysis (NRA). The samples were thinned using $\mathrm{Ga}^{+}$focused ion beam (FIB) and cleaned at low ion energy $(10 \mathrm{keV})$. TEM imaging and analysis were carried out at $200 \mathrm{keV}$ using a TECNAI F20 equipped with Gatan Energy Filter GIF 2000.

Results: Figure 1 shows an example of properties of a $300 \mathrm{~nm}$ thick $\mathrm{Fe}_{88} \mathrm{Hf}_{2} \mathrm{~N}_{10}$ sample. TEM bright field image at left top shows columnar grains morphology. Two dark field images acquired using different diffraction beam collection presented at top and middle right highlight two different grain distributions that are complementary as indicated by the color image which is a combination of the two dark field images (in green and red). Micro-diffraction patterns acquired in $\mathrm{Si}$ (100) substrate and in the three film depths marked 1, 2 and 3 are presented at bottom of figure 1. The $\mathrm{Fe}_{88} \mathrm{Hf}_{2} \mathrm{~N}_{10}$ film properties can be summarized: The bottom part (level 1 ) is quasi amorphous or very small grains BCC crystal [110] textured. The middle part (level 2) is composed of small diameter vertical BCC crystal grains [110] textured extending in the lower part but sometimes also to the top surface. The film upper part (level 3) contains large BCC grains which have a [200] texture and variable lattice parameters as shown by the broad 200 diffraction spots. HRTEM imaging confirms the [110] texture of film bottom (levels 1 and 2). The BCC lattice parameter in the whole film is around $0.294 \mathrm{~nm}$.

Figure 2 presents the magnetic properties (saturation magnetization Ms and coercive field $\mathrm{Hc}$ ) of the FeHfN thin films containing various nitrogen concentrations. Hc represents the ability of a material to follow any magnetic field fluctuation. The weaker $\mathrm{Hc}$ is, the softer the magnetic properties of the material are. Ms decreases almost linearly with an increase in the nitrogen content due to $\mathrm{N}$ atoms incorporation into Fe crystal lattice. $\mathrm{N}$ incorporation leads to lattice expansion and grain size reduction that promotes soft magnetic properties. The increase of the grain size in the top part of the $300 \mathrm{~nm}$ thick $\mathrm{Fe}_{88} \mathrm{Hf}_{2} \mathrm{~N}_{10}$ sample (Fig.1) results in high Hc. Therefore soft magnetic properties are preserved if the film thickness stays below $150 \mathrm{~nm}$. At low nitrogen content [3-10 at.\%] (Fig.2), films remain nano-crystalline and $\mathrm{Hc}$ is minimal. For large nitrogen content (>14 at.\%), films consist in grains scattered in an amorphous matrix.

Figure 3a shows for example that $\mathrm{Fe}_{82} \mathrm{Hf}_{2} \mathrm{~N}_{16}$ films are quasi amorphous and contain isolated conical grains. This amorphous-like microstructure is responsible of a weakening of the inter-granular exchange coupling leading to high $\mathrm{Hc}$ (fig.2). In such inhomogeneous films the composition variation at the nanometric scale can be controlled by STEM EELS analysis. In figure 3b, Fe and $\mathrm{N}$ concentration profiles in $\mathrm{Fe}_{82} \mathrm{Hf}_{2} \mathrm{~N}_{16}$ film are displayed. The Nitrogen concentration is roughly homogeneous but iron concentration changes of about $10 \%$ in the conical grains. These grains are $\mathrm{N}$ and (possibly) $\mathrm{Hf}$ rich.

Conclusion: Microscopy study (crystallography and chemical analysis) allows the development of FeHfN thin film with optimum soft magnetic properties for integration in silicon device in particular for inductors above IC. 


\section{References}

[1] B. Viala, M. K. Minor, and J. A. Barnard, J. Appl. Phys. 80 (1996) 3941

[2] K. Seemann, H. Leiste and V. Bekker, J. Mag. Mat. 278 (2004) 2000
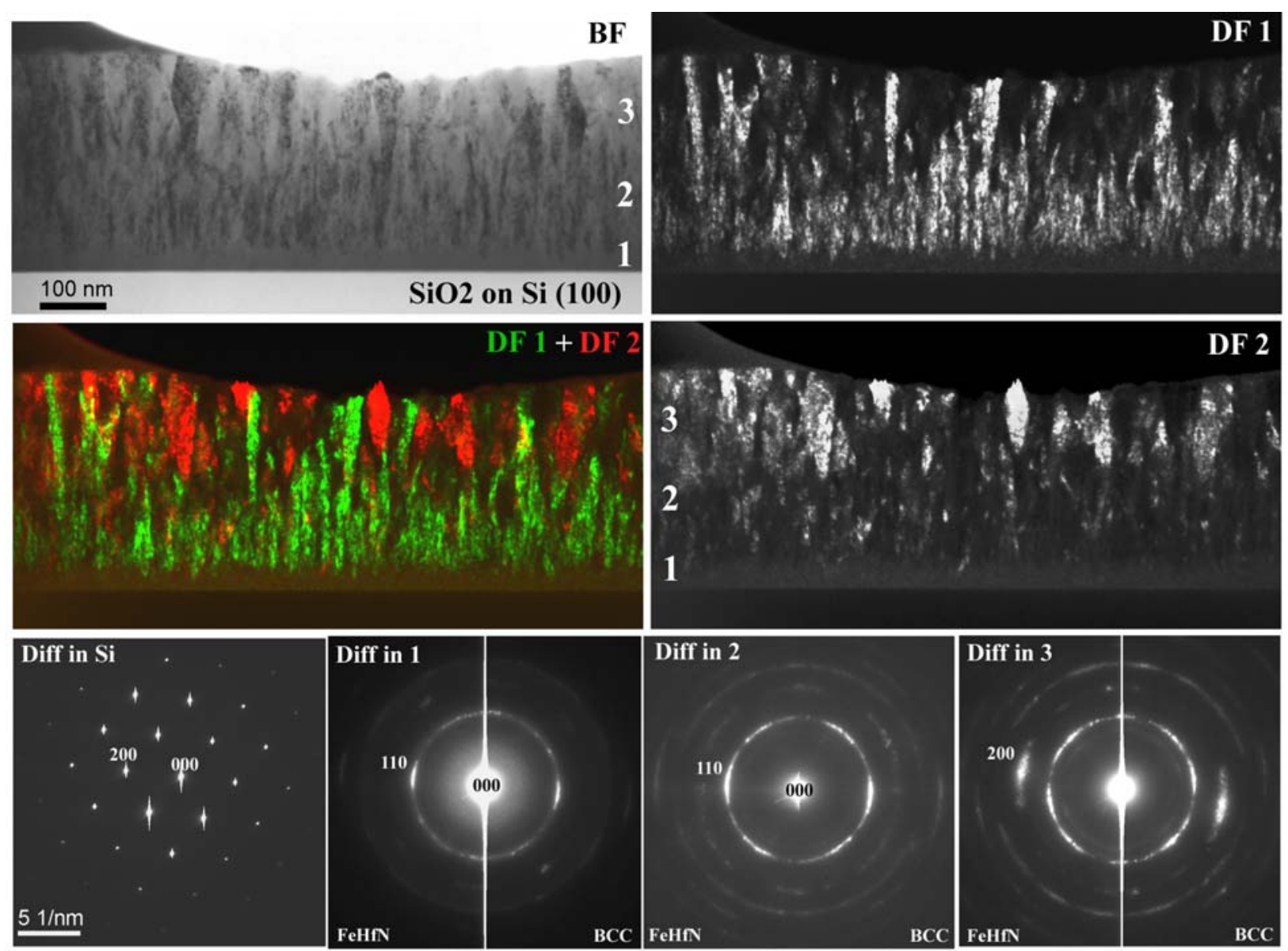

Figure 1: Top: TEM bright and dark field images of a cross section of a $\mathrm{Fe}_{88} \mathrm{Hf}_{2} \mathrm{~N}_{10}$ film $300 \mathrm{~nm}$ thick.

Bottom: micro-diffraction pattern acquired in Si (100) substrate and in FeHfN film levels marked 1, 2 and 3.

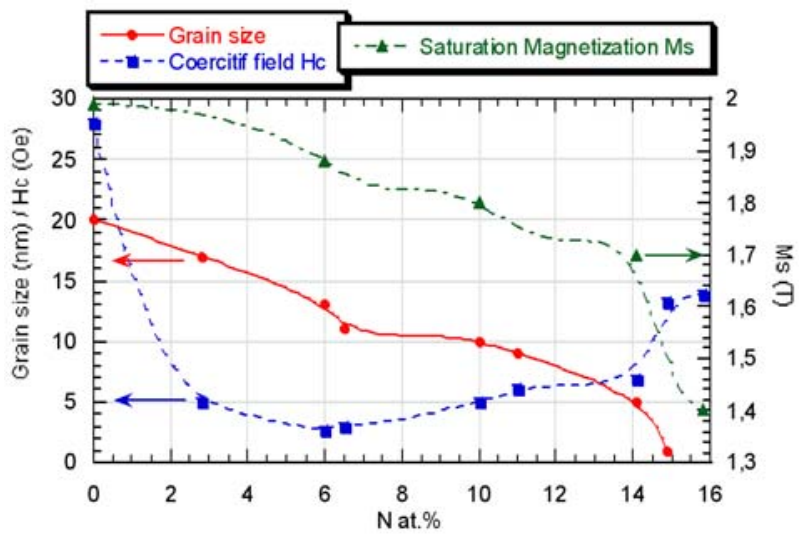

Figure 2 : Dependence of grain size, coercive field (Hc) and saturation magnetization (Ms) vs. nitrogen concentration for $100 \mathrm{~nm}$ thick FeHfN films.

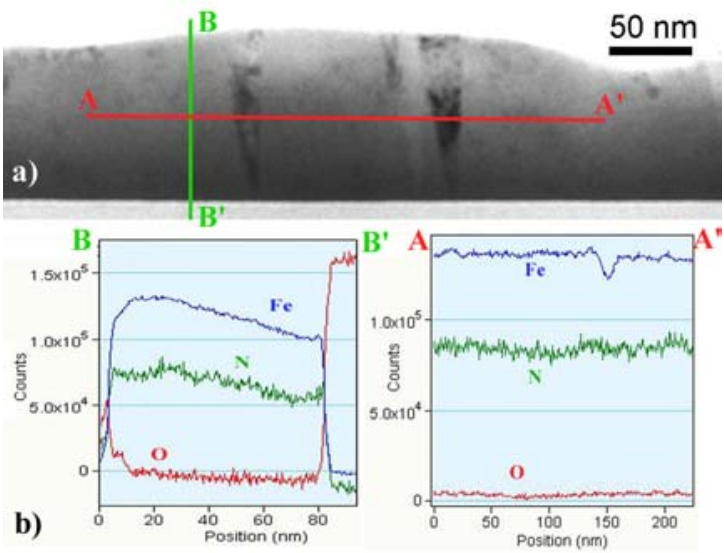

Figure 3 : a) STEM $Z$ contrast image and b) EELS line profiles analysis of a $\mathrm{Fe}_{82} \mathrm{Hf}_{2} \mathrm{~N}_{16}$ film cross section. 